\title{
Open versus Arthroscopic Treatment of Post-Traumatic Stiff Elbow
}

\author{
Ahmed Mohamed Ahmed Othman \\ Department of Orthopedic Surgery, El-Minia University, El-Minia, Egypt \\ Email: osman682012@yahoo.com
}

Received 15 September 2014; revised 2 November 2014; accepted 9 November 2014

Copyright (C) 2014 by author and Scientific Research Publishing Inc.

This work is licensed under the Creative Commons Attribution International License (CC BY). http://creativecommons.org/licenses/by/4.0/

(c) (i) Open Access

\begin{abstract}
Thirty three patients with post-traumatic stiff elbow were divided into two groups. The first group included 18 patients with an average age of 31.05 years treated by open arthrolysis. The second included 15 patients with an average age of 31.66 years treated by arthroscopic method. The results were evaluated using the Mayo clinic score and DASH score for elbow function. In the open group, after an average period of follow-up 19.33 months, the average range of flexion-extension movement improved from $51.11^{\circ}$ to $103.94^{\circ}$. The average Mayo clinic score improved from 63.38 to 92.83 and the average DASH score improved from 68.1 to 40.23 . There were 13 cases $(72.22 \%)$ excellent, 4 cases $(22.22 \%)$ good and 1 case $(5.55 \%)$ with the poor result. In the arthroscopic group, after an average period of follow-up 17.73 months, the average range of flexion-extension movement improved from $59.46^{\circ}$ to $101.53^{\circ}$. The average Mayo clinic score improved from 62.93 to 92.73 and the average DASH score improved from 69.25 to 46.4 . There were 10 cases $(66.66 \%)$ excellent, 3 cases $(20 \%)$ good and 2 cases $(13.33 \%)$ with the poor result. Both open and arthroscopic treatment can be effective in treatment of post-traumatic stiff elbow. Arthroscopic treatment is associated with less post-operative pain and morbidity. However, open treatment provides better range of motion and marked improvement of elbow function.
\end{abstract}

\section{Keywords}

Post-Traumatic Stiff Elbow, Open Arthrolysis, Arthroscopic Treatment, Mayo Clinic Performance Score, DASH Score

\section{Introduction}

The normal arc of motion of the elbow is from 0 to 145 degrees of flexion [1].

A functional arc of elbow motion between 30 and 130 degrees is necessary to complete activities of daily living, but higher demand vocational and athletic activities may require broader arcs and should be addressed ac- 
cordingly on an individualized basis [2]. Consequently, a stiff elbow has been defined as an elbow with a reduction in extension greater than 30 degrees and/or a flexion less than 120 - 130 degrees [3].

Morrey (1990) [4] classified elbow stiffness into 2 groups based on etiology and anatomic location of the pathology. Intrinsic contractures are characterized by intra-articular conditions such as degenerative osteophytes, osteochondritis dissecans, articular incongruity, loose and foreign bodies, intra-articular adhesions and synovitis. Extrinsic contractures are due to extraarticular conditions such as scarring of the capsule, collateral ligaments, musculature and skin.

Treatment of the stiff elbow begins with clinical evaluation of stiffness and identification of indications for surgical intervention [5].

Nonsurgical modalities that may be effective in improving elbow motion include physical therapy, serial casting, splinting and manipulation [6].

When conservative treatment fails to restore an acceptable range of motion, open techniques have been shown to be successful options [7].

The early application of elbow arthroscopy for treatment of stiff elbow was limited to those patients with less severe contractures or to those requiring minimal osteophytic release to improve motion [8]-[10].

With advances in the technique, arthroscopic management has supplanted open management of the stiff elbow as the operative treatment of choice in many cases. Much more extensive processes are currently treated arthroscopically, with outcomes and complication rates comparable with open releases [11] [12].

The use of arthroscopy has become more popular for several reasons including better visualization of intraarticular structures, less tissue trauma and the ability to begin early postoperative motion [7].

When performed in properly selected patients, both arthroscopic and open treatment of elbow stiffness can have satisfactory results [6].

The aim of our study was to assess clinical outcomes following treatment of posttraumatic elbow stiffness using arthroscopic arthrolysis in a group of patients compared with open arthrolysis in another group.

\section{Patients and Methods}

Between September 2008 and September 2013, 3 cases of post-traumatic stiff elbow and were divided into two groups of patients according to the method of operative treatment. The patients were divided into two groups; the first group included 18 cases (11 males and 7 females) were treated by open arthrolysis. The second group included 15 cases (10 males and 5 females) were treated by arthroscopic arthrolysis. Selection of the method of treatment was based on the method of pseudo randomization i.e. one case treated by open technique and the next case will be treated by arthroscopic technique unless there is a contraindication or limitation for arthroscopic treatment such as cases with neglected fractures or dislocations of the elbow or the head of radius or cases with marked alteration of anatomy (e.g. radial head dislocation, unreduced radial head fractures, un-united fractures...etc.) as seen in the pre-operative X-ray or CT scan are treated only by open arthrolysis after correction of the altered anatomy. Previous ulnar nerve transposition anteriorly may require open exploration to assure safe medial portal placement.

\section{Classification of Patients According to Initial Trauma}

\subsection{In the Open Group}

6 olecranon fractures, 5 radial head fractures, 2 capitellum fracture, and 2 distal humeral extra-articular fractures and 3 intercondylar fractures.

\subsection{In the Arthroscopic Group}

4 olecranon fractures, 5 radial head fractures, 1 coronoid fracture, 2 capitellum fracture, 1 fracture of the medial epicondyle and 2 distal humeral fracture.

Pre-operative heterotopic ossifications were found in 4 cases in the open group and in 2 cases of the arthroscopic group.

In the open group, the age of the patients ranged between 9 and 47 years with an average age of 31.05 years, the time to operation ranged between 6 months and 24 months with an average of 11.44 months, the pre-operative Mayo clinic score ranged from 38 to 68 with an average of 63.38 , and the pre-operative range of motion 
ranged from 38 to 68 degrees with an average of 51.11 .

In the arthroscopic group, the age of the patients ranged between 17 and 50 years with an average age of 31.66 years, the time to operation ranged between 6 months and 22 months with an average of 11.2 months, the pre-operative Mayo clinic score ranged from 57 to 71 with an average of 62.93, and the pre-operative range of motion ranged from 40 to 70 degrees with an average of 59.46 .

\section{Inclusion Criteria}

Cases with unilateral post-traumatic stiff elbow after a minimum period of conservative treatment for 6 months.

\section{Exclusion Criteria}

1) Non-traumatic causes of stiff elbow;

2) Traumatic causes associated with chronic inflammatory diseases as collagen diseases or active myositis;

3) Patients with burns;

4) Elbow instability;

5) Presence of hardware or metal implants;

6) Non-union of fractures.

\section{Pre-Operative Preparation}

All patients were preoperatively evaluated by X-ray elbow (AP, lateral, medial oblique and lateral oblique views) and if required CT scan in some cases (Table 1 and Figure 1).

\section{Technique of Open Arthrolysis (Morrey 1990) [4]}

Place the patient supine on the operating table with a sandbag under the ipsilateral shoulder. Drape the extremity free, and bring the forearm across the abdomen. Extend the Kocher approach by making an extensile skin incision $(15 \mathrm{~cm})$ proximally along the supracondylar ridge; continue it distally to the lateral epicondyle, ending over the subcutaneous border of the ulna. Proximally carry the dissection into the supracondylar ridge, with subperiosteal stripping anterior to the anterior aspect of the capsule. Distally, open the interval between the extensor carpi ulnaris and anconeus to expose the elbow joint. Place retractors deep to the extensor carpi radialis longus, brachioradialis, and brachialis. Reflect the anconeus subperiosteally off the ulna. Reflect the distal triceps off the posterior humerus and its tendon subperiosteally off the olecranon. Perform an anterior capsulectomy from lat-

Table 1. Pre-operative data.

\begin{tabular}{ccc}
\hline & Open Group & Arthroscopic Group \\
\hline Average age (Years) & $31.05(9-47)$ & $31.66(17-50)$ \\
Sex (Male/Female) & $11 / 7$ & $10 / 5$ \\
Time to operation (Months) & $11.44(6-24)$ & $11.2(6-22)$ \\
Mayo clinic score & $63.38(58-75)$ & $62.93(57-71)$ \\
Range of motion & $51.11^{\circ}(38-68)$ & $59.46^{\circ}(40-70)$ \\
\hline
\end{tabular}

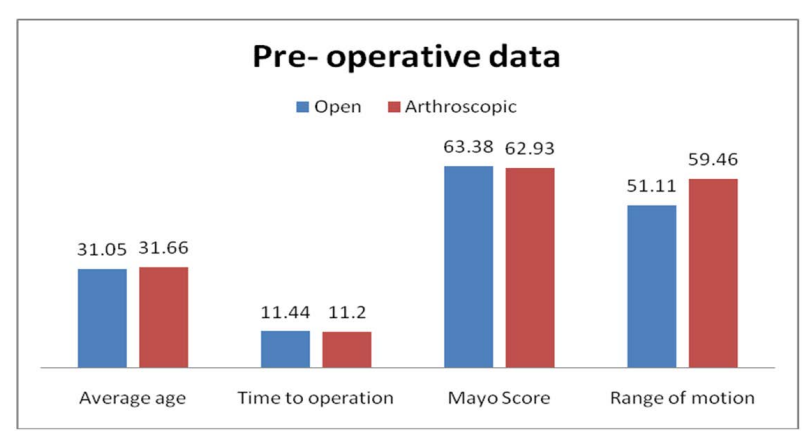

Figure 1. Pre-operative data. 
eral to medial. The lateral collateral ligament has to be reflected in a distally based flap to do this. If complete extension is not achieved, debride the olecranon fossa and the top of olecranon along with any enlargement of its margins. To improve flexion, perform a tenolysis of the triceps and a posterior capsulectomy. If flexion to 135 degrees is impossible, look for a source of anterior impingement. Remove scar tissue or heterotopic tissues in the coronoid fossa or a portion of the coronoid proximal to the brachialis insertion. Suture the lateral collateral ligament with non-absorbable sutures to its anatomical position, and carefully repair the entire lateral tissue sleeve. Apply a splint with the elbow in full extension.

\section{Rehabilitation}

A constant passive motion machine is used 12 hours a day for 1 week. After this first phase, active and passive range of motion exercises are done hourly, with the elbow splinted between exercise sessions and at night. Supervised physical therapy visits continue for 6 weeks, two or three times a week. At 6 weeks, daytime splinting is discontinued, although night splinting is continued for another 6 weeks.

\section{Arthroscopic Technique [6]}

Arthroscopy was done through the standard portals. The need for additional portals was decided intra-operatively.

The sequence for arthroscopic management of the arthrofibrotic elbow is as follows:

1) Diagnostic arthroscopy of the anterior compartment

2) Anterior debridement

a) Loose body removal;

b) Coronoid spur resection;

c) Radiocapitellar debridement.

3) Anterior capsular release with excision

a) Medial to lateral resection;

b) Expose brachialis;

c) Diagnostic arthroscopy of the posterior compartment.

5) Posterior debridement

a) Loose body removal;

b) Olecranon fossa debridement;

c) Elevate triceps with capsular release as necessary;

d) Olecranon spur resection.

6) Medial gutter debridement

7) Lateral gutter debridement

8) Olecranon fossa fenestration

The portals are closed over drain.

\section{Postoperative Management}

Initiate continuous passive motion in the recovery room set at the maximum operative motion achieved, and continue its use for 3 weeks. An aggressive stretching and strengthening program begins on the day of surgery coincides with this passive program, continuing daily for 3 weeks, and 3 times per week thereafter. Adjustable static splinting may be used after 3 weeks. If significant postoperative motion loss occurs, gentle manipulation under anesthesia to break up the early adhesions within the first 3 weeks. This subset of patients may be at increased risk for ulnar nerve dysfunction [6].

Prevention of hetrotopic ossification post-operatively was done in either technique by early motion, physical therapy and a post-operative course of NSAIDs for 4 weeks post-operatively.

\section{Results}

In the open group, the period of follow up ranged between 7 months and 39 months with an average period of 28.33 months.

Concerning the range of motion, for flexion-extension, there was an improvement from 82.3 pre-operatively 
to 123.4 degrees with an average of 103.94 degrees with a mean improvement of 41.1 degrees relative to the preoperative range $(\mathrm{p}<0.001)$.

For supination-pronation there was an improvement from an average 106 degrees pre-operatively to average 157.1 degrees at the final follow-up, with a mean improvement of 51.1 degrees relative to the preoperative range $(\mathrm{p}<0.001)$. The VAS improved from 7 (average 6 - 9) to 2.8 (average $2-5$ ).

According to Mayo clinic score [13] for elbow function, the score improved from 63.38 (range 58 - 75) to 92.83 (range 80 - 107). The DASH score improved from an average of 68.1 (range 62 - 80) to 40.23 (range 40 $45)$.

There were 13 cases (72.22\%) excellent, 4 cases (22.22\%) good and 1 case (5.55\%) with poor result.

In the arthroscopic group, the period of follow up ranged between 7 months to 31 months with an average period of 22.73 months, the flexion-extension range of motion improved from 80 to 121.1 degrees with an average of 101.1 degrees with a mean improvement of 41 degrees relative to the preoperative range $(p<0.001)$.

For supination-pronation there was an improvement from an average 104.5 degrees pre-operatively to average 150.6 degrees at late follow up with a mean improvement of 46.1 degrees relative to the preoperative range $(\mathrm{p}<$ 0.001 ). The VAS improved from 7.5 (average 6 - 9) to 3.6 (average $3-6$ ).

Post-operative Mayo clinic score improved from a range of 62.93 (average 57 - 71) to a range of 92.73 (average 85 - 101).

DASH score improved from an average of 69.25 (range 60 - 82) to 46.6 (range 42 - 56).

There were 10 cases $(66.66 \%)$ excellent, 3 cases $(20 \%)$ good and 2 cases $(13.33 \%)$ with poor result.

So, the two groups gave comparable results concerning the elbow joint function and range of motion with slightly better results in the open group at late follow-up (Table 2, Table 3, Figure 2 and Figure 3).

There were neither signs of elbow instability nor radiographic evidence of osteoarthritis progression at follow-up. Furthermore, no reported cases of post-operative heterotopic ossification at late follow up in either group.

\section{Complications of Open Release:}

1) Ulnar nerve palsy occurred in two cases one case resolved after 2 weeks and the other one resolved within 4

Table 2. Post-operative results.

\begin{tabular}{ccc}
\hline & Open Group & Arthroscopic Group \\
\hline Follow-up (Months) & $7-39(19.33)$ & $7-31(17.73)$ \\
Mayo clinic score & $80-107(92.83)$ & $85-101(92.73)$ \\
Range of motion & $98-120\left(103.94^{\circ}\right)$ & $80-120\left(101.53^{\circ}\right)$ \\
\hline
\end{tabular}

Table 3. Results of the whole study.

\begin{tabular}{ccc}
\hline & Open Group & Arthroscopic Group \\
\hline Excellent & $13(72.22 \%)$ & $10(66.66 \%)$ \\
Good & $4(22.22 \%)$ & $3(20 \%)$ \\
Poor & $1(5.55 \%)$ & $2(13.33 \%)$ \\
\hline
\end{tabular}

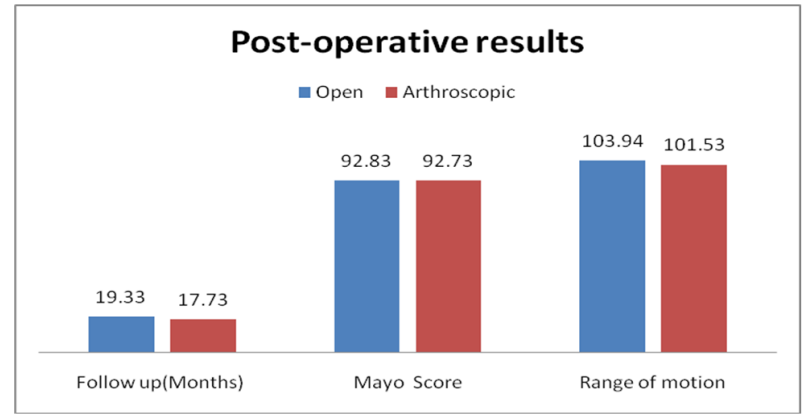

Figure 2. Post-operative results. 


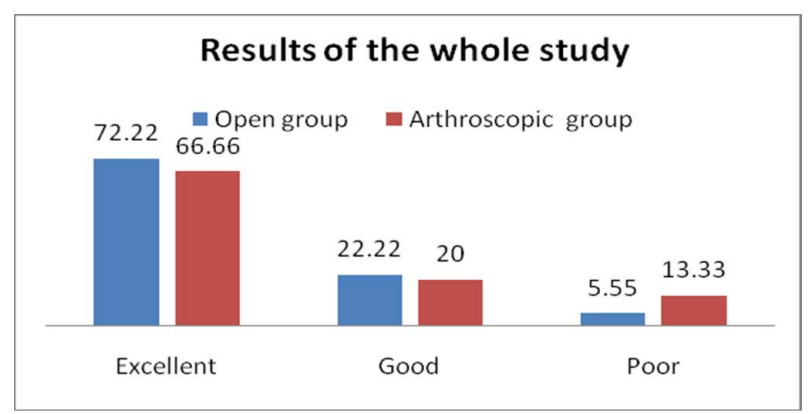

Figure 3. Results of the whole study.

weeks;

2) One case had partial wound dehiscence;

3) One had seroma resolved by using antinflammatory drugs.

None of these complications influenced the final result clinically.

\section{Complications of Arthroscopy}

1) One postoperative superficial infection of the lateral portal occurred and was successfully treated with oral antibiotics;

2) Persistent drainage from the medial portal in one case for 2 weeks which resolved by repeated dressing;

3) 2 cases of Median nerve palsy (numbness and parathesia without motor weakness) reported self improvement after 3 weeks without any residues;

4) One case with transient ulnar nerve palsy resolved after 4 weeks.

The reported complications did not affect the final outcome at the end of the study.

\section{Statistical Analysis}

The SPSS (version 11.5, SPSS Inc., Chicago, IL, USA) was used. Chi square test was used to compare the results between the two groups. The difference in the VAS was estimated using the Wilcoxon signed ranks test. Concerning the pre-operative data including the age $(\mathrm{p}=0.81607)$, pre-treatment period $(\mathrm{p}=0.873325)$, VAS ( $\mathrm{p}$ $=0.110225)$, Mayo Clinic Score $(\mathrm{p}=0.78437)$, and DASH score $(\mathrm{p}=0.31457)$, consequently, there was no significant difference. The change in the post-operative Mayo score was 29.45 for the open group $(\mathrm{p}=0.001)$ and 29.8 in the arthroscopic group $(\mathrm{p}=0.0001)$, this result was significant. The change in the post-operative DASH score was 27.87 for the open group $(p=0.001)$ and 22.85 in the arthroscopic group $(p=0.0001)$, this result was significant. The average change in the post-operative VAS was 4.2 for the open group $(p=0.001)$ and 4.1 in the arthroscopic group $(\mathrm{p}=0.0001)$. Concerning the difference in the post-operative results of both groups, there was no significant difference between the 2 groups either in the post-operative Mayo Clinic Score $(p=0.79865)$, DASH score $(\mathrm{p}=0.5402)$ or in the VAS $(\mathrm{p}$ value $=0.1579)$.

\section{Discussion}

Stiffness of the elbow is not a rare event and it can frequently lead to significant functional impairment which may be challenging to treat [14].

The goal of treatment is to obtain a low level of pain with a sufficient range of motion [15].

Release of the stiff elbow can be done by open or arthroscopic approach [16].

The literature suggests a low complication rate following open elbow capsulotomy but this data does not seem to be confirmed after a more careful reading [7].

Open elbow release with excision of tethers and blocks is a valuable procedure for post-traumatic stiffness. Recurrence in postoperative period is common but is responsive to manipulation under anesthesia and repeated releases [17].

Open release is more effective when heterotopic ossification hindering motion is removed more than when there is capsular contracture alone [18]. 
Cikes et al. (2006) [19] evaluated 18 consecutive patients at an average follow up of 16 months after open arthrolysis for posttraumatic elbow stiffness. The mean total increase in range of motion was 40 degrees, with a mean gain in flexion of 14 degrees and 26 degrees in extension.

Husband and Hastings (1990) [20] used lateral approach to release a post-traumatic contracture of the elbow in seven patients, and the results were evaluated an average of thirty-eight months postoperatively. The average arc of motion increased 46 degrees.

Tan et al. (2006) [17] in a retrospective review of 52 cases of patients who underwent open surgical treatment for post-traumatic elbow contracture. Surgery was performed at an average of 14 months from the time of injury. Follow-up was 18.7 months. The average extension-flexion arc of motion improved from 57 to 116 degrees and forearm rotation improved from 119 to 145 degrees postoperatively.

Ayadi et al. (2011) [21] performed open elbow arthrolysis in 30 cases. At final follow-up, the average arc of flexion-extension was 95 degrees $+/-15$ degrees (average flexion 120 degrees $+/-13$ degrees, average flexion contracture 31 degrees $+/-6$ degrees), with a mean improvement of 51 degrees relative to the preoperative range $(\mathrm{p}<0.001)$. The average arc of forearm rotation at final follow-up was 151 degrees $+/-23$ degrees, with a mean improvement of 41 degrees $(p<0.05)$. The average VAS was 5/10, with 6 excellent, 6 good, 6 fair and 4 poor results. The average DASH score was 31.6.

In our series, the results of open release were comparable to those achieved by other authors regarding elbow function, patient satisfaction and pain relief.

For many years open capsular release had been the standard treatment for elbow contractures. More recently the use of arthroscopy has become more popular for several reasons including better visualization of intra-articular structures, less tissue trauma from open incisions, and potentially the ability to begin early postoperative motion [22]-[24].

Limited skin incisions and soft tissue dissection not only decrease the risk of scarring, but also allow the patient to safely undertake an immediate aggressive postoperative physical therapy program. These measures reduce the recurrence of contracture in a joint well recognized for its propensity for stiffness [6].

Arthroscopy allows the surgeon to address extrinsic capsular and collateral ligament contractures, as well as intrinsic joint pathology, with increased visualization, and therefore, a more comprehensive appreciation of the pathology [6] [25].

However, arthroscopic capsular release is a technically demanding procedure that requires meticulous attention to details and should only be attempted by surgeons with extensive experience in elbow arthroscopy [26].

Recognition of the proximity of the neurovascular structures is critical to minimizing complications, but expertise in elbow arthroscopy and meticulous surgical technique can produce excellent results [6].

Ball et al. (2002) [27] reported a retrospective series of 14 patients all affected by post-traumatic elbow contracture and were treated arthroscopically. The minimum follow up was of 1 year (12 - 29 months). The mean arc of motion gain was of 41.5. At the last follow up the average pain level measured on a VAS was 3.25, the ASES functional ability score for the elbow improved in all patients, with an average score of $28.3(25-30)$ out of 30 .

Cefo and Eygendaal (2011) [28] evaluated 27 patients at 3, 12, and 24 months after arthroscopic capsular release of posttraumatic stiff elbow. Before the arthroscopic procedure, the mean flexion was $123^{\circ}\left(\mathrm{SD} 8^{\circ}\right)$, extension was $24^{\circ}\left(\mathrm{SD} 9^{\circ}\right)$, and total ROM was $99^{\circ}\left(\mathrm{SD} 11^{\circ}\right)$, and after surgery, flexion improved significantly to $133^{\circ}$ $\left(\mathrm{SD} 5^{\circ}\right)$, extension to $7^{\circ}\left(\mathrm{SD} 6^{\circ}\right)$, and total ROM to $125^{\circ}$ (SD $10^{\circ}$ ). The mean (SD) EFA showed improvement from 69 (SD 4) preoperatively to 91 (SD 4) postoperatively.

In the current series, the results of arthroscopic release match the results of other authors in terms of the range of motion, pain relief and patient satisfaction.

In comparing open and arthroscopic results for the treatment of elbow stiffness, it appears that both techniques can achieve satisfactory results when employed properly [25].

Cohen et al. (2000) [29] were the first to actually compare open and arthroscopic technique. They reported that open debridement is superior to arthroscopic fenestration of the olecranon fosse for improving range of motion. In their report, only 8 of 26 arthroscopic debridement patients showed a mean gain of $4^{\circ}$ in elbow flexion, whereas 12 of 16 patients who underwent the open procedure showed a mean gain of $15^{\circ}$ in elbow flexion. The arthroscopic procedure yielded a better pain relief.

Kodde et al. (2013) [16] Performed a comprehensive literature search. Thirty articles were included, with an overall enrolment of 798 patients treated between 1989 and 2012. There were 4 different kinds of treatment 
modalities: 1) open arthrolysis; 2) arthroscopic arthrolysis; 3) open arthrolysis with external fixation; 4) open arthrolysis with distraction arthroplasty. The gain in range of motion was $51^{\circ}, 40^{\circ}, 88^{\circ}$, and $56^{\circ}$ for groups $1-4$, respectively. The average percentage of complications was $23,5,73$, and 58 for groups $1-4$, respectively. They concluded that current literature is not sufficient enough to draw firm statistically based conclusions.

In our study, there was no significant difference in the results of both groups at the end of the study.

The reported complications for elbow arthroscopy include compartment syndrome, septic arthritis, superficial infection, persistent drainage from portal sites, and most frequently, nerve injuries (transient or permanent). Comparing complication rates between open and arthroscopic capsular release is difficult since there are no direct comparative studies in the literature [24] [30].

In our study, the reported complications did not affect the results in either group at late follow-up.

\section{Conclusion}

Both open and arthroscopic treatments are effective in treatment of post-traumatic stiff elbow. Arthroscopic treatment is associated with less post-operative pain and morbidity. However, open treatment provides better range of motion, marked improvement of elbow function and more patient satisfaction. Open treatment is more suitable for patients with disturbed anatomy as it is safer and less technically demanding than arthroscopic method. The best results were obtained in younger patients who had operation within the first year after initial trauma. Better outcome of open arthrolysis may be explained by more extensive release and the ability to slide down the tissues more than that achieved by the arthroscopic method. Poor results may be due to post-operative pain or late interference.

\section{References}

[1] O’Driscoll, G., Yamaguchi, K., King, S. and McKee, M. (2007) Arthroscopic Osteocapsular Arthroplasty. In: Advanced Reconstruction Elbow, Vol. 16, American Academy of Orthopaedic Surgeons, Rosemont.

[2] Morrey, B.F., Askew, L.J., An, K.N. and. Chao, E.Y. (1981) A Biomechanical Study of Normal Functional Elbow Motion. Journal of Bone and Joint Surgery-Series A, 63, 872-877.

[3] Søjbjerg, J.O. (1996) The Stiff Elbow. Acta Orthopaedica Scandinavica, 67, 626-631. http://dx.doi.org/10.3109/17453679608997771

[4] Morrey, B.F. (1990) Post-Traumatic Contracture of the Elbow. Operative Treatment, Including Distraction Arthroplasty. Journal of Bone and Joint Surgery (A), 72, 601-618.

[5] Jupiter, J.B., O'Driscoll, S.W. and Cohen, M.S. (2003) The Assessment and Management of the Stiff Elbow. Instructionalcourse lectures, 52, 93-111.

[6] Peden, J.P. and Savoie, F.H. (2006) Arthroscopy of the Stiff Elbow. Techniques in Orthopaedics, 21, 331-339. http://dx.doi.org/10.1097/01.bto.0000252138.82801.e1

[7] Blonna, D., Bellato, E., Marini, E., Scelsi, M. and Castoldi, F. (2011) Arthroscopic Treatment of Stiff Elbow. International Scholarly Research Network, 2011, Article ID: 378135, 7 p.

[8] Nowicki, K.D. and Shall, L.M. (1992) Arthroscopic Release of a Posttraumatic Flexion Contracture in the Elbow: A Case Report and Review of the Literature. Arthroscopy, 8, 544-547. http://dx.doi.org/10.1016/0749-8063(92)90024-6

[9] Jones, G.S. and Savoie, F.H. (1993) Arthroscopic Capsular Release of Flexion Contractures (Arthrofibrosis) of the Elbow. Arthroscopy, 9, 277-283. http://dx.doi.org/10.1016/S0749-8063(05)80422-0

[10] Strobel, M.J., Eckardt, O.A. and Eichhorn, H.J. (2001) Arthroscopic Therapy in Limited Mobility of the Elbow Joint. Der Orthopäde, 30, 610-618. http://dx.doi.org/10.1007/s001320170048

[11] Redden, J.F. and Stanley, D. (1993) Arthroscopic Fenestration of the Olecranon Fossa in the Treatment of Osteoarthritis of the Elbow. Arthroscopy, 9, 14-16. http://dx.doi.org/10.1016/S0749-8063(05)80337-8

[12] McLaughlin II, R.E., Savoie, F.H., Field, L.D. and Ramsey, J.R. (2006) Arthroscopic Treatment of the Arthritic Elbow Due to Primary Radiocapitellar Arthritis. Arthroscopy, 22, 63-69. http://dx.doi.org/10.1016/j.arthro.2005.10.013

[13] Morrey, B., An, K. and Chao, E. (1993) Functional Evaluation of the Elbow. In: Morley, B.F., Ed., The Elbow and Its Disorders, 2nd Edition, WB Saunders, Philadelphia, 86-97.

[14] Nandi, S., Maschke, S., Evans, P.J. and Lawton, J.N. (2009) The Stiff Elbow. Journal of Hand Surgery, 4, 368-379.

[15] Chammas, M. (2014) Post-Traumatic Osteoarthritis of the Elbow. Orthopaedics and Traumatology: Surgery \& Research, 100, S15-S24.

[16] Kodde, I.F., van Rijn, J., van den Bekerom, M.P. and Eygendaal, D. (2013) Surgical Treatment of Post-Traumatic El- 
bow Stiffness: A Systematic Review. Journal of Shoulder and Elbow Surgery, 22, 574-580. http://dx.doi.org/10.1016/j.jse.2012.11.010

[17] Tan, V., Daluiski, A., Simic, P. and Hotchkiss, R.N. (2006) Outcome of Open Release for Post-Traumatic Elbow Stiffness. Journal of Trauma, 61, 673-678. http://dx.doi.org/10.1097/01.ta.0000196000.96056.51

[18] Lindenhovius, A.L., Linzel, D.S., Doornberg, J.N., Ring, D.C. and Jupiter, J.B. (2007) Comparison of Elbow Contracture Release in Elbows with and without Heterotopic Ossification Restricting Motion. Journal of Shoulder and Elbow Surgery, 16, 621-625. http://dx.doi.org/10.1016/j.jse.2007.01.005

[19] Cikes, A., Jolles, B.M. and Farron, A. (2006) Open Elbow Arthrolysis for Posttraumatic Elbow Stiffness. Journal of Orthopaedic Trauma, 20, 405-409. http://dx.doi.org/10.1097/00005131-200607000-00007

[20] Husband, J.B. and Hastings II, H. (1990) The Lateral Approach for Operative Release of Post-Traumatic Contracture of the Elbow. Journal of Bone and Joint Surgery. American Volume, 72, 1353-1358.

[21] Ayadi, D., Etienne, P., Burny, F. and Schuind, F. (2011) Results of Open Arthrolysis for Elbow Stiffness. A Series of 22 Cases. Acta Orthopaedica Belgica, 77, 453-457.

[22] Kim, S.J. and Shin, S.J. (2000) Arthroscopic Treatment for Limitation of Motion of the Elbow. Clinical Orthopaedics and Related Research, 375, 140-148. http://dx.doi.org/10.1097/00003086-200006000-00017

[23] Nguyen, D., Proper, W., MacDermid, S.I.J.C., King, G.J.W. and Faber, K.J. (2006) Functional Outcomes of Arthroscopic Capsular Release of the Elbow. Arthroscopy, 22, 842-849. http://dx.doi.org/10.1016/j.arthro.2006.04.100

[24] Nathan, L., Van Zeeland, N.L. and Yamaguchi, K. (2010) Arthroscopic Capsular Release of the Elbow. Journal of Shoulder and Elbow Surgery, 19, 13-19. http://dx.doi.org/10.1016/j.jse.2010.01.003

[25] Pederzini, L., Tosi, M., Prandini, M. and Nicoletta, F. (2013) Elbow Arthroscopy in Stiff Elbow. In: Elbow Arthroscopy, Springer, Berlin, 43-56. http://dx.doi.org/10.1007/978-3-642-38103-4 5

[26] Noojin, F., Savoie, F. and Field, L. (2001) Arthroscopic Release of the Stiff Elbow. Techniques in Shoulder \& Elbow Surgery, 2, 17-25. http://dx.doi.org/10.1097/00132589-200103000-00003

[27] Ball, C.M., Meunier, M., Galatz, L.M., Calfee, R. and Yamaguchi, K. (2002) Arthroscopic Treatment of Post-Traumatic Elbow Contracture. Journal of Shoulder and Elbow Surgery, 11, 624-629. http://dx.doi.org/10.1067/mse.2002.126770

[28] Cefo, I. and Eygendaal, D. (2011) Arthroscopic Arthrolysis for Posttraumatic Elbow Stiffness. Journal of Shoulder and Elbow Surgery, 20, 434-439. http://dx.doi.org/10.1016/i.jse.2010.11.018

[29] Cohen, A.P., Redden, J.F. and Stanley, D. (2000) Treatment of Osteoarthritis of the Elbow: A Comparison of Open and Arthroscopic Debridement. Arthroscopy, 16, 701-706. http://dx.doi.org/10.1053/jars.2000.8952

[30] Kelly, E.W., Morrey, B.F. and O’Driscoll, S.W. (2001) Complications of Elbow Arthroscopy. Journal of Bone and Joint Surgery. American Volume, 83, 25-35. 
Scientific Research Publishing (SCIRP) is one of the largest Open Access journal publishers. It is currently publishing more than 200 open access, online, peer-reviewed journals covering a wide range of academic disciplines. SCIRP serves the worldwide academic communities and contributes to the progress and application of science with its publication.

Other selected journals from SCIRP are listed as below. Submit your manuscript to us via either submit@scirp.org or Online Submission Portal.
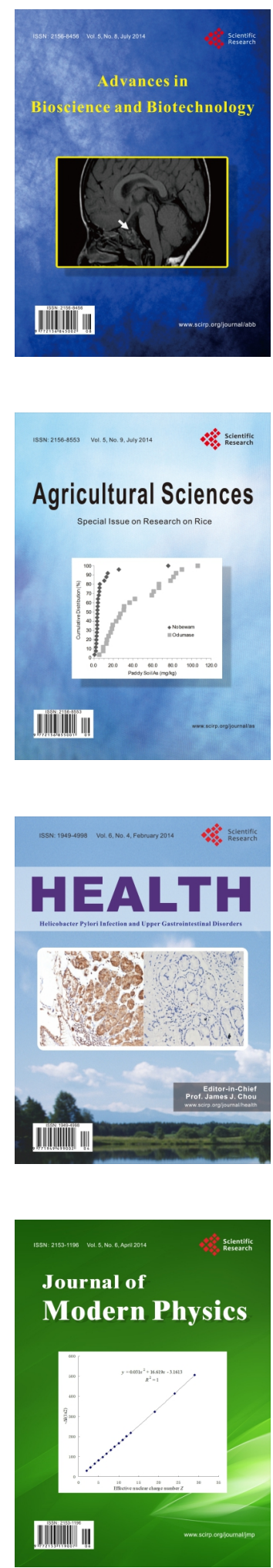
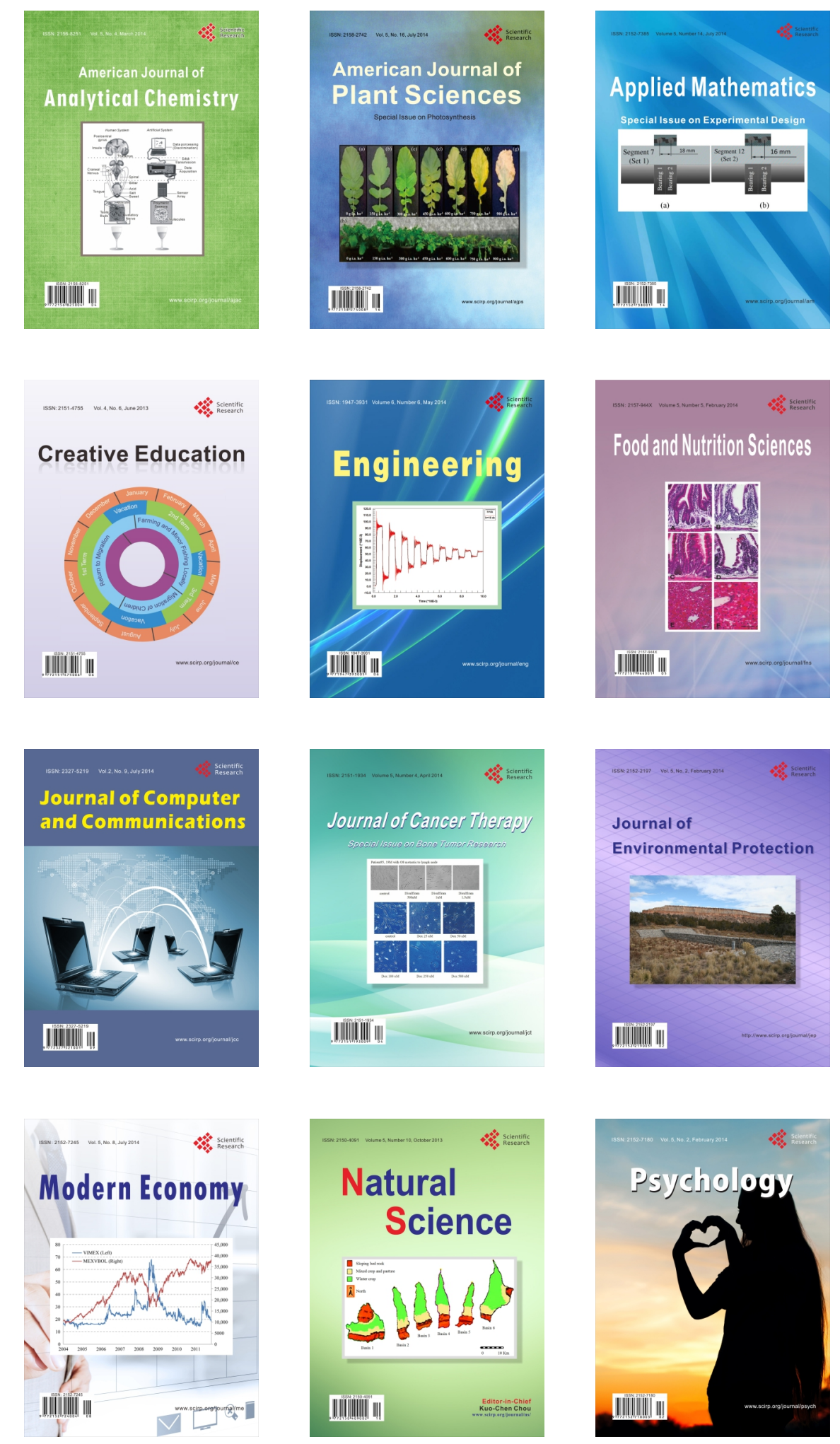